\title{
Erratum: Scaling considerations in ground-state quantum computation [Phys. Rev. A 65, 022315 (2002)]
}

Ari Mizel, M. W. Mitchell, and Marvin L. Cohen

(Received 22 July 2020; published 26 October 2020)

DOI: 10.1103/PhysRevA.102.049905

We have become aware of errors in the proof of the lower bound on the gap, presented in Sec. II. Most importantly, it is not correct to apply Eq. (4) to the case of an arbitrary computer considered in Sec. II C, so Eq. (13) does not follow from Eq. (12). In addition, the CNOT Hamiltonian $h_{A, B}^{j}\left(\right.$ CNOT) needs to be replaced with $h_{A, B}^{j}(1$, CNOT) of Eq. (3) of Ref. [1]. This replacement is needed because the extra penalty terms in Eq. (3) of Ref. [1] push up extraneous undesired ground states. With this correction, one expects the $\sim 1 /(N+1)^{4}$ lower bound stated in Eq. (14) to still hold. Indeed, although the proof in our paper is not correct, such a lower bound is proven in Theorems 4 and 5 of a recent publication [2] for a mildly modified Hamiltonian with some circuit restrictions detailed there. Another proof of a polynomial lower bound on the gap, requiring somewhat different Hamiltonian modifications and restrictions, appears in Ref. [3]. The conclusions of our paper remain unchanged.

[1] A. Mizel, D. A. Lidar, and M. W. Mitchell, Phys. Rev. Lett. 99, 070502 (2007).

[2] A. Mizel, Phys. Rev. A 99, 022311 (2019).

[3] N. P. Breuckmann and B. M. Terhal, J. Phys. A: Math. Theor. 47, 195304 (2014). 